\title{
ATUAÇÃO DA EQUIPE DE ENFERMAGEM AOS PACIENTES VÍTIMAS DE ACIDENTES DE TRÂNSITO
}

\author{
ROLE OF THE NURSING TEAM WITH PATIENTS WHO ARE VICTIMS \\ OF TRAFFIC ACCIDENTS
}

\author{
Ana Luiza Macedo Dias ${ }^{1}$ \\ Andreza Correia de Oliveira ${ }^{2}$ \\ José Maxwell de Araújo Silva ${ }^{3}$ \\ Maria Danielly Benício de Araújo ${ }^{4}$ \\ Paloma Emanuelle dos Santos ${ }^{5}$
} Renata Lívia Silva Fonsêca Moreira de Medeiros ${ }^{6}$

RESUMO: OBJETIVO: identificar quais as condutas da equipe de enfermagem frente ao paciente vítima de acidentes de trânsito. METODOLOGIA: o presente estudo constitui-se de um método de revisão integrativa da literatura, buscando artigos na Biblioteca Virtual de Saúde (BVS) voltada para a atuação da equipe de enfermagem aos pacientes vítimas de acidentes de trânsito, utilizando os descritores assistência pré-hospitalar, acidentes de trânsito e cuidados de enfermagem. RESULTADOS: após análise de 45 artigos, foram excluídas 41 publicações por não atenderem o objetivo do trabalho, como resultado compôs o estudo, 04 artigos. Nele comprovou que o perfil epidemiológico da população do estudo destacou a predominância do sexo masculino com faixa etária de 15 a 44 anos, decorrentes de imprudências no trânsito nas quais o resultado Traumatismo Crânioencefálico (TCE) é uma das principais causas de morte, possuindo o nordeste como região destaque entre os artigos analisados e que a atuação da enfermagem é indispensável na prestação da assistência, tomando decisões rápidas e precisas fazendo com que o

\footnotetext{
1 Graduanda pela Universidade Federal de Campina Grande - UFCG. Email: analuizamacedodias@gmail.com;

2 Graduada pela Faculdade São Francisco da Paraíba - FASP. Email: andrezalavras123@hotmail.com;

3 Graduado pela Faculdade Santa Maria - FSM. Email: maax2015.1@gmail.com;

4 Graduada pela Universidade Federal de Campina Grande - UFCG. E-mail: daniellybenicio@outlook.com;

5 Graduada pela Universidade Federal de Campina Grande - UFCG. E-mail: palomae.santos@gmail.com;

${ }^{6}$ Docente da Faculdade Santa Maria-FSM. Enfermeira pela Universidade Federal da Paraíba-UFPB. Especialista em Saúde Pública pela FACISA. Mestre em Enfermagem pela-UFPB. Doutora em Pesquisa em Cirurgia pela Faculdade de Ciências Médicas Santa Casa de São Paulo - FCMSP. Email: renaliviamoreira@hotmail.com.
} 
risco de lesões, traumatismos e risco de morte seja o mínimo possível para as vítimas acometidas em acidentes traumáticos. CONCLUSÃO: constatou-se no trabalho a importância de desenvolver medidas educativas no trânsito, através de ações dentro e fora do ambiente hospitalar, trabalhando de forma integral e multidisciplinar entre profissionais, pacientes e a sociedade no geral. Conclui-se com este trabalho que os profissionais necessitam estar qualificados de forma rotineira para que a atuação frente ao paciente vítima de acidentes automobilísticos seja realizada de forma eficaz, portanto, contribuindo para o sucesso do atendimento, diminuindo as sequelas e riscos à vida.

Decs: Assistência pré-hospitalar; acidentes de trânsito; cuidados de enfermagem.

ABSTRACT: OBJECTIVE: to identify the behavior of the nursing team towards patients who are victims of traffic accidents. METHODOLOGY: the present study consists of an integrative literature review method, seeking articles in the virtual health library (VHL) focused on the performance of the nursing staff to patients victims of traffic accidents, using the descriptors pre-hospital care, traffic accidents and nursing care. RESULTS: After analyzing 45 articles, 41 publications were excluded for not meeting the objective of the work, as a result composed the study 04 articles. It proved that the epidemiological profile of the study population highlighted the predominance of males aged 15 to 44 years, resulting from imprudence in traffic in which the result of traumatic brain injury (TBI) is one of the main causes of death, with the northeastern as a highlighted region among the articles analyzed and that the role of nursing is essential in providing assistance, making quick and accurate decisions, making the risk of injuries, trauma and risk of death as little as possible for victims affected in traumatic accidents. CONCLUSION: It was verified in the work the importance of developing educational measures in traffic, through actions inside and outside the hospital environment, working in an integral and multidisciplinary way among professionals, patients and society in general. It is concluded with this work that professionals need to be routinely qualified so that the work in front of the patient victim of car accidents is carried out effectively, therefore, contributing to the success of the service, reducing the consequences and risks to life.

Descriptors: Pre-hospital care; traffic-accidents; nursing care. 


\section{INTRODUÇÃO}

Os Acidentes de Trânsito (AT) estão entre as causas externas que possuem elevadas taxas de morbimortalidade, sendo um problema na saúde pública e mundial, fazem parte de um grupo de eventos e agravos à saúde que são consideradas epidemias que envolvem naturalmente os pedestres, ciclistas, motociclistas, ocupantes de carro de pequeno porte, caminhonete ou veículo de transporte pesado, causando vítimas no Brasil e no mundo (SILVA, 2012).

Os AT correspondem à cerca de 1,35 milhão de mortes por ano em todo o mundo, sendo uma das principais causas de lesões e mortes entre pessoas em faixa etária de 5 a 29 anos (WHO, 2018). O Brasil encontra-se entre os países que apresentam mais de $60 \%$ das mortes por acidentes, caracterizando o perfil dos acidentes comprova-se que o meio de transporte mais identificado durante os infortúnios no trânsito é a motocicleta (DA SILVA DANTAS, 2017). Em concordância, - Ministério da Saúde no ano de 2018 salientou que no nordeste brasileiro as internações por causas externas forma de aproximadamente 25 mil vítimas (SANTOS et al. 2021). O que acarreta em consequências sociais e econômicas a população.

Diante desse cenário, em 29 de setembro de 2003, foi instituído na Política Nacional de Atenção as Urgências através da Portaria n 1.863/GM, a implantação dos Serviços de Atendimento Móvel de Urgências (SAMU 192) nos municípios. Dentre os serviços de Atendimento Pré-hospitalar (APH), destaca o SAMU 192, que tem como objetivo chegar precocemente à vítima, após um agravo à saúde de natureza clínica, cirúrgica, traumática, pediátrica, obstétrica e psiquiátrica que podem acarretar sofrimento, sequelas ou até mesmo à morte do paciente. O SAMU 192 é um serviço gratuito que funciona 24 horas, por meio de veículos tripulados por médicos, enfermeiros, auxiliares de enfermagem e condutores socorristas que são acionados pela Central de Regulação de Urgências (BESSA, 2018). 
Existe a necessidade de uma equipe multiprofissional qualificada para diminuir os danos e riscos de agravamento ao quadro do paciente, o enfermeiro no $\mathrm{APH}$, assume o papel de articulador incorporado à equipe, consistindo em ser o elo da relação entre os diversos atores da assistência multiprofissional (BESSA, 2018). O profissional enfermeiro estabelece assim, a ligação entre assistência, gestão, coordenação do serviço e a equipe de enfermagem.

Este estudo justifica-se pela necessidade de aprofundar o conhecimento sobre os acidentes de trânsito, suas vítimas e identificar os cuidados da equipe de enfermagem frente aos traumas, assim como determinar estimativas e analisar a distribuição espacial das taxas de mortalidade. Dessa forma, este trabalho objetiva descrever a atuação da equipe de enfermagem ao paciente vítima de acidentes de trânsito.

\section{METODOLOGIA}

Utilizou-se o método de revisão integrativa da literatura que segundo Mendes, Silveira e Galvão, (2008) inclui a análise e síntese de pesquisas publicadas possibilitando conclusões referentes às determinadas áreas de estudos. Portanto, esse tipo de metodologia oportuniza aos profissionais da enfermagem, a realização de uma análise critica dos conteúdos.

A revisão contará com as seguintes etapas: 1) definição da pergunta norteadora; 2) delineamentos dos critérios de inclusão e exclusão da pesquisa; 3) avaliação dos estudos incluídos na amostra; 4) interpretação independente dos resultados obtidos; 5) análise e apresentação dos resultados.

Para guiar a revisão integrativa, foi formulada a seguinte pergunta norteadora: Quais são as condutas da equipe de enfermagem frente ao paciente vítima de acidente trânsito?

Para a seleção dos artigos utilizou-se a Biblioteca Virtual de Saúde (BVS), as bases de dados utilizadas foram as seguintes: Literatura Latino-Americana e do Caribe em Ciências da Saúde (LILACS), Medical Literature Analysis and Retrieval 
Sistem (MEDLINE), Bases de Dados em Enfermagem (BDENF). Com uso de descritores controlados, a saber: Assistência pré-hospitalar and acidentes de trânsito and cuidados de enfermagem.

Os parâmetros de elegibilidade que complementaram a questão de pesquisa tiveram como critérios de inclusão: artigos disponíveis na íntegra de forma eletrônica, que abordaram informações sobre o tema, o período mínimo de seguimento de análise (estudos publicados entre 2011 a 2021); e Português como idioma padrão. Já os critérios de exclusão envolveram a não aceitação de dados não publicados ou em andamento, artigos em que não identificou a relação com a temática, resumos e duplicidade entre as bases de dados.

Durante a busca, identificaram-se 45 artigos ao todo na BVS, sendo 31 na MEDLINE, 11 na LILACS, 10 na BDENF e 01 na IBECS. Após análise, excluíram-se 41 por não terem correspondido à questão proposta pelo estudo. Desta forma, a amostra final foi de 04 artigos, os quais foram lidos na íntegra de forma minuciosa. Conforme é apresentado na Figura 1. 
Descritores utilizados: Assistência pré-hospitalar and acidentes de trânsito and cuidados de enfermagem.

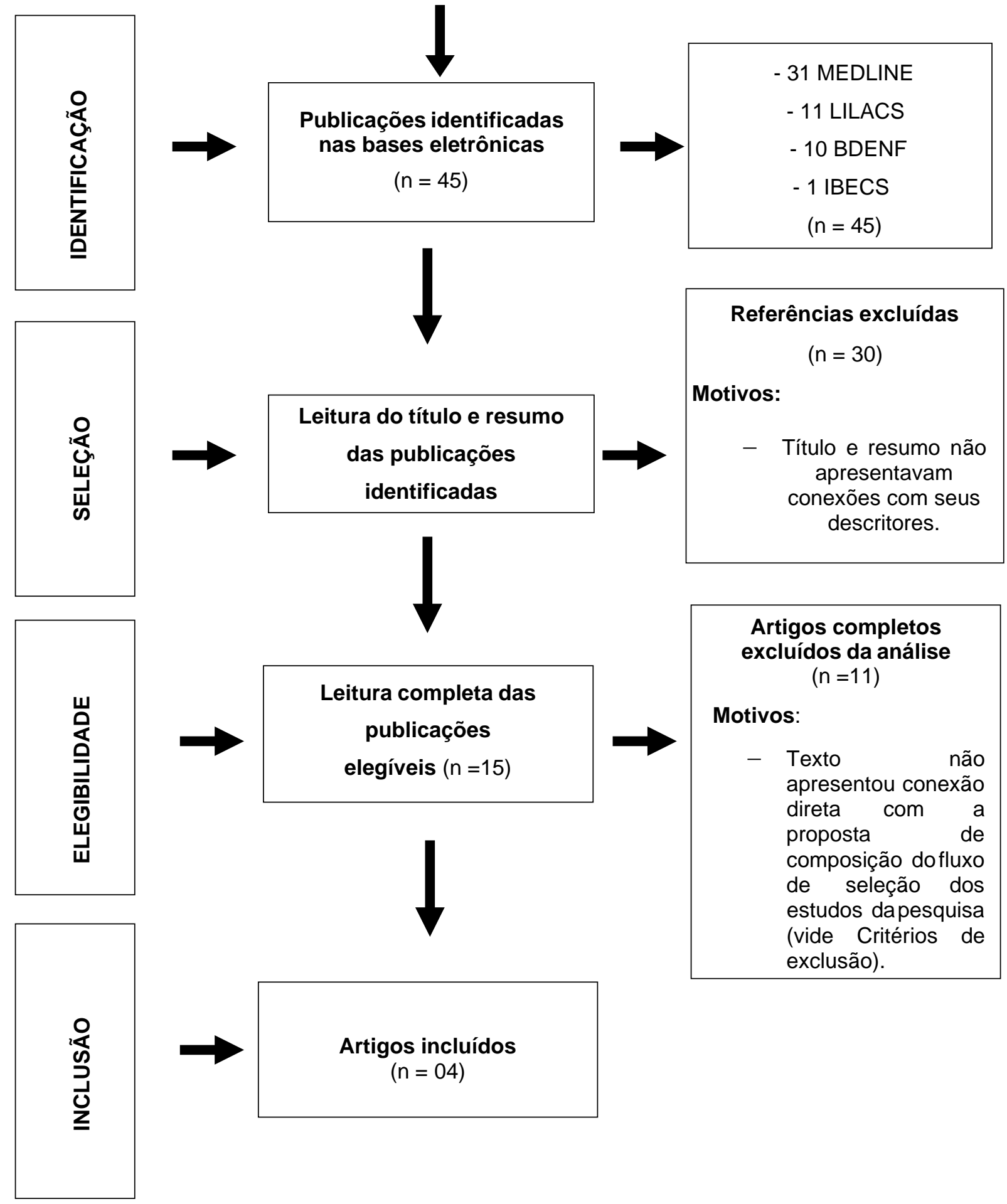




\section{RESULTADOS}

Foram encontrados 45 artigos nas bases de dados consultadas. Destes, quatro artigos preencheram os critérios de inclusão previamente estabelecidos por estarem relacionados com a temática e o objetivo deste estudo descrito.

A síntese dos estudos compreendidos neste presente trabalho foram lidos na íntegra e as informações contidas nos mesmos foram estruturadas quanto às suas características como: fonte, ano, título, autor, tipo de estudo e resultados. Observase que os trabalhos abordados na amostra foram publicados entre os anos de 2011 a 2020.

A maioria dos artigos encontrados foram da Enfermagem. As amostras tiveram em sua maioria as fichas dos usuários e fichas de SAMU. Observou-se que $25 \%$ das pesquisas encontradas fazem parte da revista do Estado do Rio de Janeiro, $50 \%$ do estado do Pernambuco e $25 \%$ Rio Grande do Norte, as quais valorizaram estudos sobre acidentes de trânsitos. Predominando os estudos na região nordeste.

Quanto ao ano, cerca de $50 \%$ dos estudos foram publicados entre os anos de 2012 a 2016 e 75\% entre os anos de 2017 a 2020. No que diz respeito aos títulos dos trabalhos, a maior parte apresenta conteúdos sobre acidentes de trânsito ou assistência de enfermagem. Em relação ao tipo de estudo, predominou-se do tipo exploratório descritivo com $100 \%$ das análises.

No que se concerne ao resultado, constata-se que a população mais acometida nos acidentes são do sexo masculino com faixa etária entre 25 a 34 anos, decorrentes de imprudências no trânsito, nas quais o Traumatismo Crânioencefálico (TCE) é uma das principais causas de mortes e/ou sequelas pelo fato dos motociclistas não utilizarem o uso do capacete como meio de proteção, associado ao excesso de velocidade. 
Tabela 1. Características gerais dos estudos sobre a atuação da equipe de enfermagem ao paciente vítima de acidentes de trânsito. Os dados presentes estão estruturados quanto a: fonte e ano, título do trabalho, autor, tipo de estudo e resultados dos artigos analisados.

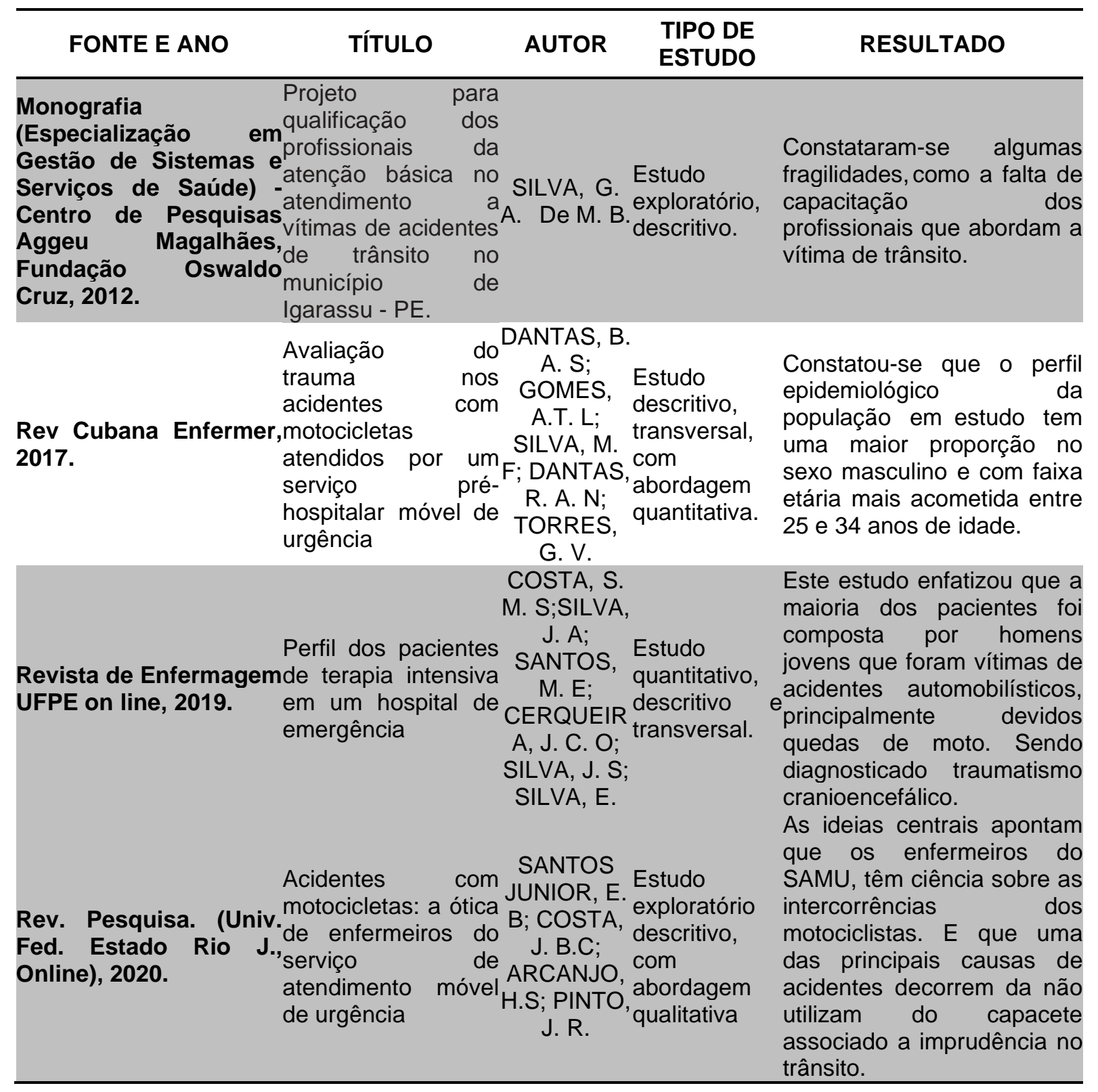




\section{DISCUSSÃO}

De acordo com Dantas et al (2017) e Costa et al (2019) a grande maioria dos acidentes automobilísticos acometem com mais incidência o público masculino jovem, com faixa etária de 15 a 44 anos, visto que os condutores possuem comportamentos mais agressivos ao dirigirem quanto comparados em relação às mulheres. Associa-se também com fatores de risco tais como: ausência do uso de capacete e materiais de proteção, excesso de velocidade e alcoolemia.

Nesse sentindo, Dantas et al (2017), Costa et al (2019) e Santos Junior (2020) complementam afirmando que quando se compara as ocorrências e o horário que ocorrem, observa-se registros dos acidentes automobilísticos no período noturno, associado às festas e comemorações nas quais, possui ingestão alcoólica exacerbadamente sem moderação por jovens levando a quedas de motos e colisões fatais durante a madrugada.

Para Santos Junior (2020) a atuação da enfermagem é indispensável, sendo o enfermeiro mediador da assistência deve atuar de forma organizada e sistemática, tomando decisões rápidas e precisas, fazendo com que o risco de lesões, traumatismos e risco de morte sejam o mínimo possível para as vítimas acometidas em acidentes traumáticos. Por conseguinte,é fundamental a atualização profissional em relação aos cuidados prestados perante os pacientes atendidos em serviços de APH móvel.

Os achados nos estudos de Silva (2012) e Costa et al (2019) corroboram que o que mais acomete os profissionais atuantes frente às vitimas de acidentes de trânsito é a falta de qualificação profissional, com isso gera-se o sentimento de incapacidade, ocasionando um atendimento ineficaz. Portanto, é imprescindível a capacitação profissional para que a assistência ao politraumatizado seja realizada de forma íntegra e eficiente.

Conforme entendimento de Silva (2012) e Santos Junior (2020) ações preventivas de acidentes automobilísticos, assim como: panfletagem, palestras, jogos educativos, softwares e obediência à sinalização do trânsito, são fundamentais 
para diminuirem o índice de lesões e mortes ocasionadas pelo trauma. Mas, para que isso se torne necessário é importante o envolvimento de profissionais da saúde, educadores e da população em geral.

\section{CONSIDERAÇÕES FINAIS}

A caracterização do perfil epidemiológico da população do estudo destacou a predominância do sexo masculino com faixa etária de 15 a 44 anos nos traumas automobilísticos com motocicletas, associando-se a ingestão de bebida alcoólica e imprudência no trânsito.

Ressalta-se também que perfil da região analisada abordado pela pesquisa indica o nordeste como a de destaque entre os artigos analisados, o que pode servir como exemplo para futuras pesquisas e ações para conscientizar a população quanto às medidas preventivas.

Constatou-se no trabalho a importância de desenvolver medidas educativas no trânsito, através de ações dentro e fora do ambiente hospitalar, trabalhando de forma integral e multidisciplinar entre profissionais, pacientes e a sociedade no geral.

Conclui-se com este trabalho que os profissionais necessitam estar qualificados de forma rotineira para que a atuação frente ao paciente vítima de acidentes automobilísticos sejarealizada de forma eficaz, portanto, contribuindo para o sucesso do atendimento, diminuindo as sequelas e riscos à vida. 


\section{REFERÊNCIAS BIBLIOGRÁFICAS}

BESSA, S. L. Assistência de enfermagem ao paciente politraumatizado nas Unidades de Atendimento Móveis. Porto Velho, 2018.2 Disponível em: <http://repositorio.saolucas.edu.br:8080/xmlui/bitstream/handle/123456789/2758/Bessa\%2C\%20 Simone\%20Lorena\%20da\%20Silva\%20-

\%20Assist\%C3\%AAncia\%20de\%20enfermagem\%20ao\%20paciente\%20politraumatizado\%20nas $\% 20$ unidades $\% 20$ de $\% 20$ atendimento $\% 20 \mathrm{~m} \% \mathrm{C} 3 \%$ B3veis.pdf? sequence $=1$ \&isAllowed $=\mathrm{y}>$ acesso em 25de ago de 2021.

COSTA, Sara Maria et al. Perfil dos pacientes de terapia intensiva em um hospital de emergência. Revista de Enfermagem UFPE on line, [S.I.], v. 13, nov. 2019. ISSN 1981-8963. Disponível

em: <https://periodicos.ufpe.br/revistas/revistaenfermagem/article/view/242359/33884>. Acessoem: 31 jul. 2021.

DA SILVA DANTAS, Bruno Araújo et al. Avaliação do trauma nos acidentes com motocicletas atendidos por um serviço pré-hospitalar móvel de urgência. Rev CubanaEnfermer, Ciudad de la Habana, v.33, n.2, jun. 2017.2 Disponível em: <http://scielo.sld.cu/scielo.php?script=sci_arttext\&pid=S0864-

$03192017000200007 \&$ Ing=es\&nrm=iso>. Acesso em 31 jul. 2021.

DAVIM, Rejane Marie Barbosa et al. Urgências e emergências: do atendimento pré-hospitalar aos cuidados intensivos. Revista de Enfermagem UFPE on line, [S.I.], v. 10, n. 11, p. 41574160, set. 2016. Disponível em: <https://periodicos.ufpe.br/revistas/revistaenfermagem/article/view/11504>. Acesso em: 31 jul. 2021.

MENDES, Karina Dal Sasso; SILVEIRA, Renata Cristina de Campos Pereira; GALVÃO, Cristina Maria. Revisão integrativa: método de pesquisa para a incorporação de evidências nasaúde e na enfermagem. Texto \& contexto-enfermagem, v. 17, p. 758-764, 2008. Disponível em: < https://www.scielo.br/j/tce/a/XzFkq6tjWs4wHNqNjKJLkXQ/abstract/?lang=pt> Acesso em 19 de ago de 2021.

SANTOS JUNIOR, E. B; et al. Acidentes com motocicletas: a ótica de enfermeiros do serviçode atendimento móvel de urgência. Rev. Pesqui. (Univ. Fed. Estado Rio J., Online), jan.-dez. 2020. Disponível em <http://seer.unirio.br/index.php/cuidadofundamental/article/view/8324/pdf> Acesso em 31jul. 2021.

SANTOS, J. J. S. et al. Epidemiologia das vítimas de trauma atendidas por serviço préhospitalar. Rev. Pesqui. (Univ. Fed. Estado Rio J., Online), jan.-dez. 2021. Disponível em: < http://seer.unirio.br/index.php/cuidadofundamental/article/view/8563/pdf_1>. Acesso em 31 jul. 2021.

SILVA, G. A. de M. B. Projeto para Qualificação dos Profissionais da Atenção Básica no Atendimento a Vítimas de Acidentes de Trânsito no Município de Igarassu - PE. Recife, 2012.

WORLD HEALTH ORGANIZATION. Global status report on road safety 2018. Genebra:World Health Organization; 2018. Disponível em: <https://www.who.int/violence_injury_prevention/road_safety_status/2018/en/> acesso em $22 \mathrm{de}$ ago de 2021. 